\title{
Performance Study of Passivity Based Control on Heat Exchanger Networks
}

\author{
Varintorn Athithunchaiyaphong ${ }^{1}$, Niaon Prajimtis ${ }^{2}$, Thongchai Srinophakun ${ }^{2}$ \\ ${ }^{1}$ Center of Excellence on Petrochemical and Materials Technology \\ Pathumwan, Bangkok, Thailand \\ ${ }^{2}$ Department of Chemical Engineering, Faculty of Engineering, Kasetsart University \\ Bangkhen, Bangkok, Thailand \\ fengtcs@ku.ac.th
}

\section{Extended Abstract}

Heat exchanger networks (HEN) are major consumption units which are energy efficient and economical in the chemical and processing industry [1]. The design of heat exchanger networks is a subject that has received significant attention for quite some time. One of the methods for optimal design of heat exchanger networks is the passivity concept [2] which is the analysis of the general process stability that can be used as a powerful tool in analysing dynamic systems and controller design [3]. This concept is implemented to guarantee controllability and robust controller tuning. Thus, this work mainly focuses on the application of a passivity method for heat exchanger networks of the Ethyl Benzene process [4] and to perform an analysis of a network cost index after the process improvement by the Aspen Energy Analyzer. Dynamic models are generated for all variables of the unit in the state space domain. Subsequently, the state space model of heat exchanger networks is formulated to study the concept of passivity and is analysed in terms of transfer functions, while the control structure is analysed by linear matrix inequality. Then, these transfer functions are analysed by the passivity index to indicate that the heat exchanger networks are passive or not. In addition, the presence of passivity behaviour is dependent upon its possible pairing schemes. Therefore, the magnitude of the passivity index was used to rank the pairing schemes. Consequently, the passivity based decentralized unconditional stability PI controllers for this system were designed and verified with the Aspen Dynamics Simulator. Moreover, the control performance was tested with a set point temperature and disturbance changes by the comparison of both auto and passivity tunes. The results show that the analysis of an Ethyl benzene plant with the heat exchanger networks by using the Aspen Energy Analyzer has a total cost of $3.764 \times 103$ cost/years or $98.3 \%$ of the target. As part of the control system, it was found that the paring of scheme 1 (1$1 / 2-2 / 3-3 / 4-4)$ is the best pairing. This pairing scheme provided the largest frequency bandwidth and did not require an offset at all. Although the set point and disturbance has been changed, scheme 1 presented good results. Furthermore, this scheme includes four controllers which are TCC1, TCC2, TCH1 and TCH2. The response of these controllers for passivity based control reach the set point temperature very smoothly at $0.3,0.1,3.2$ and 4.7 hours, respectively. On the other hand, the auto PI control for these controllers reach the set point temperature at $1.5,1.5$, and 0.75 for TCC1, TCC2 and TCH1 respectively. The set point temperature was not reached for TCH2. In conclusion, the passive controllers outperform the auto PI tuning controllers.

\section{References}

[1] H. Wu, F. Yan, W. Li and J. Zhang, "Simultaneous heat exchanger network synthesis involving nonisothermal mixing streams with temperature-dependent heat capacity," Ind. Eng. Chem. Res., vol. 54, no. 36, pp. 8979-8987, 2015.

[2] J. Bao and P. L. Lee, Process Control: The Passivity System Approach. London: Springer-Verlag, 2007.

[3] V. Ghanbari, M. Xia and P. Antsalkis, "A passivation method for design of switched control," in American Control Conference, Boston, MA, pp. 1572-1577, 2016.

[4] W. Luyben, "Ethyl Benzene process," in Plantwide Dynamic Simulators in Chemical Processing and Control, Bethlehem, PA: Marcel Dekker, Inc., pp. 160-178, 2002. 